\title{
Parasitic Leiomyoma- Peritoneum- A Rare Case
}

\author{
Dr. Purva V Reelkar ${ }^{1 *}$, Dr. Vijay V Reelkar ${ }^{2}$
}

${ }^{1}$ MS OBGY, Shraddha Hospital, Chiplun, Dist- Ratnagiri, Maharashtra, India

${ }^{2}$ MD OBGY DGO, Shraddha Hospital, Chiplun, Dist- Ratnagiri, Maharashtra, India

DOI: $10.36347 /$ simcr.2021.v09i04.001

| Received: 05.03.2021 | Accepted: 23.03.2021 | Published: 02.04.2021

*Corresponding Author: Dr. Purva V Reelkar

Abstract

When uterine fibroid occurs in its usual site, presentation, diagnosis and management are straightforward. When it exists in unusual area, the diagnosis becomes challenging. Parasitic leiomyoma is an extremely rare disease. Their unusual growth pattern may even mimic malignancy and can result in clinical dilemma. A 42 years old female with complaints of lump in abdomen since 4 years and amenorhoea since 3 months, was having USG and CT scan findings consistent with heterogenous mass arising from uterus. Intraoperatively, peritoneal soft tissue tumour was found and HPE confirmed the diagnosis of parasitic leiomyoma.

Keywords: Leiomyoma, Parasitic, Myomectomy, Fibroid.

Copyright $\left({ }_{0} 2021\right.$ The Author(s): This is an open-access article distributed under the terms of the Creative Commons Attribution 4.0 International License (CC BY-NC 4.0) which permits unrestricted use, distribution, and reproduction in any medium for non-commercial use provided the original author and source are credited.

\section{INTRODUCTION}

Uterine leiomyoma is the most common tumor affecting woman's genital tract. By the age of 35 years, at least $20 \%$ of females develop uterine fibroid. When it occurs in its usual site, presentation, diagnosis and management are straightforward. When it exists in unusual area, the diagnosis becomes challenging [1]. Although extremely rare, any anatomical site might be involved. The etiology is unknown, however iatrogenic cause is suggested [2]. Several authors reported parasitic leiomyoma occurring after laparoscopic removal of fibroid or myomectomy. It can be explained by unintentional seeding of the fragments during the procedure [3]. Parasitic leiomyoma is an extremely rare disease. Their unusual growth pattern may even mimic malignancy and can result in clinical dilemma. Subserosal fibroids are located underneath the mucosal (peritoneal) surface of the uterus and can become very large and grow out in a papillary manner to become pedunculated fibroids. If a pedunculated subserosal fibroid develops an extremely long stalk, it is called wandering or migrating leiomyoma. These pedunculated growths can occasionally detach from the uterus to become a parasitic leiomyoma [4].

\section{CaSe Report}

A 42 years old, married, house wife presented with complaints of lump in abdomen since 4 years and amenorrhea for 3 months. Family, drug, and past medical and surgical history were negative. Vital signs were normal. Abdominal examination revealed a mass of 16 - 18 weeks size, palpable more towards the left side occupying left lumbar and left iliac fossa. The mass was variable in consistency and mobile in all directions. Overlying skin was normal. Bi-manual examination confirmed the mass to be separate from the uterus. Ultrasound examination revealed a heterogeneous mass, $15 \mathrm{~cm}$ in diameter in the midline, located cephalad to the uterus and urinary bladder. The uterus was found to be bulky with multiple fibroids. CT scan revealed the same heterogeneous mass consisting of solid and predominantly cystic elements situated antero-superior to the bladder. A provisional diagnosis of ovarian tumor or pedunculated subserosal fibroid was made and she was planned for laparotomy. Intra-operatively she was found to have a mass of $15 \times 13 \mathrm{~cm}$ beneath the rectus muscle and above the parietal peritoneum extending laterally from the midline on the left side. Sharp dissection was performed and the plane of cleavage was obtained. The mass was variable in consistency. The mass was excised in toto. We proceeded to Total Abdominal Hysterectomy and she was found to have bulky uterus with multiple fibroids. Incidentally, a small right ovarian cyst of $3 \times 3 \mathrm{~cm}$ was also noted. The anterior parietal wall mass weighed 600 grams and was thought to be a soft tissue tumor. The specimen was sent for histopathological examination and the findings were consistent with leiomyoma. On her follow-up visit, she was doing well with no recurrence of mass or pain abdomen. 


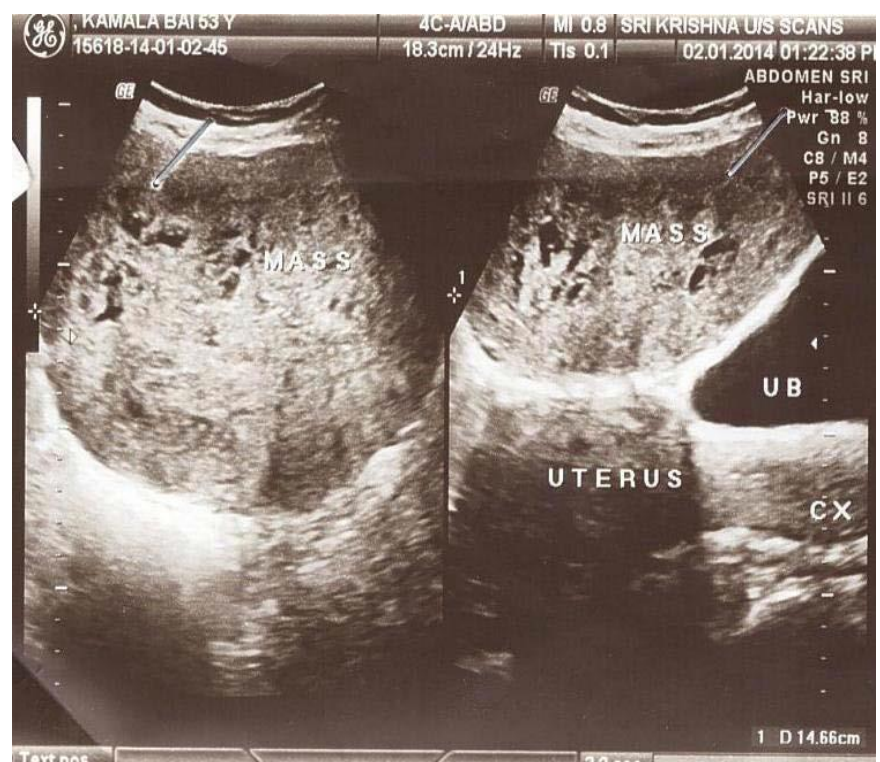

USG image showing the relation of the uterus and the mass
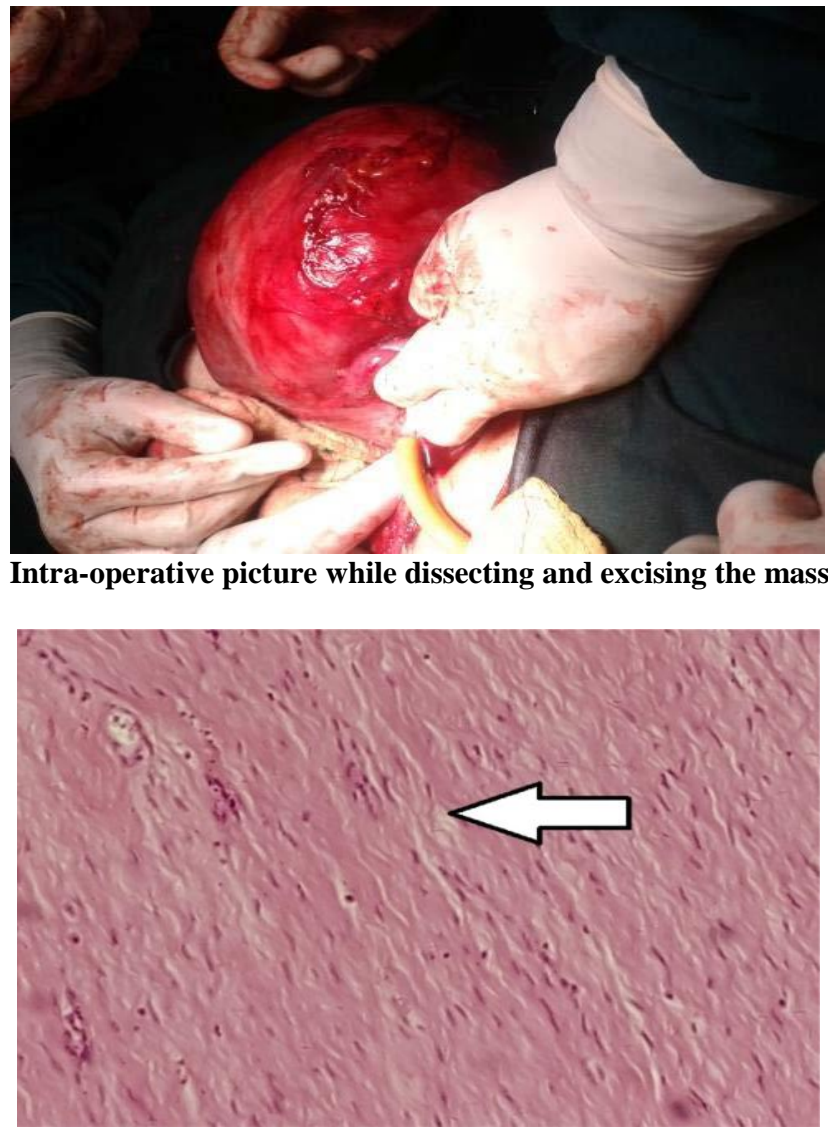

Whorled pattern of smooth muscle bundle separated by well vascularized connective tissue (white arrow)

\section{DISCUSSION}

Uterine fibroids are the most common benign pelvic tumors in women and are present in about $80 \%$ of all hysterectomy specimens [5]. The most common sites for fibroid are uterus and gastrointestinal tract. However, they are known to originate from wherever smooth muscle cells exist [6]. Whether smooth muscle cells in vessels of anterior abdominal wall and peritoneum react to the extraneous hormonal stimulation to form leiomyoma is yet to be explained. The term Parasitic Leiomyoma was first coined by Kelly and Cullen in 1909 and they could either be:

1. Primary or spontaneous, explained as pedunculated subserosal fibroid which develops a long stalk, outgrowing their uterine blood supply and subsequently receiving blood supply from other sources or

2. Secondary or iatrogenic, seeding a portion of the fibroid during morcellation and leaving 
behind a small fragment that implants to the normal tissue anywhere in the peritoneum [7, 8].

In a study by Kho and Nezhat, twelve cases were studied and they reported $83 \%$ of patients had prior abdominal surgery and $67 \%$ patients had prior myomectomy [9].

Our patient did not have any prior surgical history and we wished to hypothesize that the leiomyoma was one of the parasitic wondering varieties. With the setting of multiple fibroids in this patient's uterus, we could also conclude that this was a parasitic fibroid rather than a de novo leiomyoma arising in non Mullerian smooth muscle of the peritoneal cavity or abdominal wall. Our case reiterates the important fact that parasitic leiomyomas present with diagnostic dilemmas for even astute clinician as they often masquerade as adnexal masses.

Conflicts of interest: There is no conflict to be declared.

Funding: No source to be stated

Ethical approval: Approval has been taken from bioscience centre.

Consent: Written consent has been taken from the patient for publication of this report.

\section{REFERENCES}

1. Grover A, Bhalla S. Parasitic leiomyoma: a rare complication following laparoscopic myomectomy with review of literature. Curr Med Res Pract. 2015;5(6):278-281.

2. Iida M, Ishikawa H, Shozu M. Spontaneous parasitic leiomyoma in a post-partum woman. J Obstet Gynaecol Res. 2016;42(12):1874-1877.

3. Moon HS, Koo JS, Park SH, Park GS, Choi JG, Kim SG. Parasitic leiomyoma in the abdominal wall after laparoscopic myomectomy. Fertility and sterility. 2008 Oct 1;90(4):1201-e1.

4. Robbins Clotran RS. Pathologic Basis of Diseases. 1984 3rd Edition, Philadelphia, 1109.

5. Cramer SF, Patel A. The frequency of uterine leiomyomas. American journal of clinical pathology. 1990 Oct 1;94(4):435-8.

6. Watanabe K, Tanaka M, Kusakabe T, Soeda S. Mesometrial smooth muscle as an origin of female retroperitoneal (pelvic) leiomyomas. Virchows Archiv. 2007 Nov;451(5):899-904.

7. Paal E, Miettinen M. Retroperitoneal Leiomyomas-A Clinicopathologic and Immunohistochemical Study. American Journal of Clinical Pathology, 2001; 25:1355-1363.

8. Kumar S, Sharma JB, Verma D, Gupta P, Roy K. Disseminated Peritoneal Leiomyomatosis. Archives of Gynecology and Obstetrics, 2008;278:93-95.

9. Kho KA, Nezhat C. Parasitic myomas. Obstetrics \& Gynecology. 2009 Sep 1;114(3):611-5. 